\title{
Clinical and financial impacts of introducing an endoscopic mucosal resection service for treatment of patients with large colonic polyps into a regional tertiary hospital
}

\section{(ㄷ)(우우}

\author{
Authors \\ Thomas Worland ${ }^{1}$, Oliver Cronin², Benjamin Harrison², Linda Alexander ${ }^{3}$, Nik Ding ${ }^{4,5}$, Alvin Ting ${ }^{2,6}$, Stephanie \\ Dimopoulos $^{6}$, Racheal Sykes ${ }^{6}$, Sina Alexander ${ }^{2,6}$
}

\author{
Institutions \\ 1 Monash Health, Melbourne, Australia \\ 2 University Hospital Geelong, Geelong, Australia \\ 3 The University of Notre Dame, Fremantle, Australia \\ 4 St Vincent's Hospital, Melbourne, Australia \\ 5 University of Melbourne, Melbourne, Australia \\ 6 Deakin University, Geelong, Australia
}

submitted 12.1.2019

accepted after revision 17.6.2019

\author{
Bibliography \\ DOI https://doi.org/10.1055/a-0970-8828 | \\ Endoscopy International Open 2019; 07: E1386-E1392 \\ (c) Georg Thieme Verlag KG Stuttgart · New York \\ eISSN 2196-9736
}

Corresponding author

Dr. Sina Alexander, Department of Gastroenterology, Barwon Health, University Hospital Geelong, Ryrie St., Geelong, Australia 3220

sinaa@barwonhealth.org.au

\section{ABSTRACT}

Background and study aims Endoscopic mucosal resection (EMR) of large sessile or laterally spreading colonic lesions is a safe alternative to surgery. We assessed reduc- tions in Surgical Resection (SR) rates and associated clinical and financial benefits following the introduction of an EMR service to a large regional center.

Patients and methods Ongoing prospective intention-totreat analysis of EMR was undertaken from time of service inception in 2009 to 2017. Retrospective data for SR of large sessile/laterally spreading colonic lesions were collected for the period 4 years before commencement of the EMR service (2005 - 2008) and 9 years after its introduction (2009-2017).

Results From 2005 to 2008, 32 surgical procedures were performed for non-malignant colonic neoplasia (50\% male, median age 68 years, median Length of Stay (LoS) 10 days). Following the introduction of the EMR service, there was a $56 \%$ reduction in the number of patients referred for surgery (32 surgical procedures, $47 \%$ male, median age 70 years, median LoS 8.5 days). During this period, EMR was successfully performed in 183 patients with 216 lesions resected ( $60 \%$ male, median age 68 years, median LoS 1 day). Compared to the SR group, the EMR cohort had a lower peri-procedural complication rate $(7.7 \%$ vs $54.7 \%, P<$ 0.0001 ), and shorter average LoS ( 1 vs 9 days, $P<0.0001$ ). A cost saving of AUD $\$ 19543.5$ was seen per lesion removed with EMR compared to SR.

Conclusions The introduction of a dedicated EMR service into a large regional center as an alternative to SR can lead to a substantial decrease in unnecessary surgery with subsequent clinical and financial benefits.

\section{Introduction}

Endoscopic mucosal resection (EMR) undertaken via colonoscopy is a technique developed for the treatment of large sessile or laterally spreading colonic lesions and has proved to be a safe and effective alternative to surgery [1]. These precursors to colorectal cancer (CRC) are seen in up to $5.2 \%$ of colonoscopies [2] and have traditionally been managed surgically. Endoscopic submucosal dissection (ESD), developed in the East, is an emerging alternative modality to surgery and EMR, but is not routinely available in the West [3]. With expertly trained endos- copists, lesions up to $100 \mathrm{~mm}$ can be removed efficiently by EMR and most recurrences can easily be managed endoscopically [4].

EMR of mucosal neoplastic lesions or Submucosal layer 1 $(\mathrm{Sm} 1)$ cancers results in the same outcomes as surgical resection with low recurrence rates and the advantage of far lower associated morbidity and mortality [5, 6]. The safety of EMR has been well defined with a complication rate of $4-7 \%$, most commonly manifesting as peri-procedural bleeding [7-9]. These complication rates are less than those associated with laparoscopic or open surgical procedures $(19-21 \%)[10,11]$. 
Open colorectal surgery is associated with a postoperative recovery that mandates an inpatient stay of up to 13.2 days whereas EMR procedures are routinely performed as day cases [10]. Peri-procedural mortality following elective colonic resection is $1.38-1.56 \%$ [12], compared with EMR mortality of $0 \%$ $[5,13]$.

Many large tertiary centers have developed advanced endoscopy units providing patients with the option of EMR in preference to operative management of appropriate colonic lesions. In a large Australian multicenter trial, 401 of 478 patients (84\%) with large lesions avoided the need for surgery by using EMR [13]. With a growing body of evidence for its safety, EMR is becoming an effective alternative to traditional operative management and there is now a strong impetus to provide greater access to EMR to a broader population. No significant difference in rates of complete excision, complications, and recurrences has been found between EMR performed at high versus low volume centers [9]. Despite the findings of increased efficiency, reduced costs and decreased morbidity and mortality of EMR, surgery continues to be undertaken for a large number of endoscopically appropriate lesions [14-16].

Geelong, a major Australian regional center located in Victoria, had an EMR service introduced in 2009. This study aimed to analyze the safety, success rate, and reduction in invasive surgery rate associated with establishing this regional EMR service. Secondary aims were to assess mortality and morbidity rates and the financial implications of the introduction of this EMR service.

\section{Materials and methods}

\section{Study design and inclusion criteria}

This is an ongoing prospective observational cohort study inclusive of all consecutive adult patients with colorectal lesions referred for EMR from the time of service inception in January 2009 to December 2017. The study was approved by the University Hospital Geelong Human Research Ethics Committee (reference number 12/140). Exclusion criteria included patients referred for EMR with lesions less than $15 \mathrm{~mm}$ that were resected by traditional hot/cold snare polypectomy; lesions that were below the dentate line; or lesions involving the appendiceal orifice or ileocecal valve. Patients with previous incomplete resections performed at our or other institutions were also included. EMR was not attempted in lesions with features strongly suggestive of submucosal invasion. In these instances, surgical management was recommended. All lesions were initially identified by consultant endoscopists from Geelong and the surrounding referring area. In addition, data were collected retrospectively for all patients who underwent SR for benign colorectal lesions in the period 4 years before the introduction of the EMR service (2005 to 2008) as well as in the subsequent 9 years (2009 to 2017).

\section{Data collection}

Data collected included patient demographics, lesion characteristics including size, location, and histology, EMR or type of surgery performed, complications, length of stay (LoS), recur- rence rates, peri-procedural mortality, and Weighted Inlier Equivalent Separation (WIES) values. If a patient required readmission in the immediate post-procedural period for a related complication, the LoS and WIES values of this second admission were included as part of the index procedure admission totals. The senior investigator completed detailed data sheets for each EMR patient and these, along with results, complications and follow-up colonoscopies were recorded in a database by a dedicated research assistant. Surgical data were abstracted from a hospital database and reviewed by two researchers. In Victorian public hospitals, inpatient encounters are funded according to activity-based case mix via WIES. Higher WIES values are assigned to more costly inpatient stays with the state government providing funding accordingly. At the time of writing, the monetary funding for one WIES point was AUD \$4545.

\section{Procedures}

Colonoscopy was performed using Olympus 180 series variable-stiffness colonoscopes (Olympus Optical, Japan, Tokyo) with intravenous sedation. Split-dose bowel preparation was used in the majority of cases and colonic insufflation with carbon dioxide was introduced soon after commencement of the study. Both white light and narrow-band imaging (NBI), with or without distal cap attachment, were used to assess each lesion.

All EMR procedures were performed as day cases by the senior investigator. The diameter of the lesion was estimated at the time of colonoscopy by reference to an open snare placed adjacent to the lesion. Technical success was defined as complete removal of all visible neoplastic tissue in a single treatment session. EMR was performed by the "sequential inject and resect" technique [17]. A solution consisting of $1 \mathrm{~mL}$ of $0.4 \%$ Indigo Carmine or Methylene Blue and $1 \mathrm{~mL}$ of $1: 10000$ epinephrine combined with $8 \mathrm{~mL}$ of $0.9 \%$ saline solution was injected into the submucosal layer under the lesion to separate the mucosa from its underlying muscularis propria for safe resection. Resection was performed predominantly using a 20$\mathrm{mm}$ sized spiral snare and microprocessor controlled electrosurgical generator. En bloc resection was attempted for most lesions $<25 \mathrm{~mm}$. For lesions $>25 \mathrm{~mm}$, sequential piecemeal EMR resection was performed, with care to ensure the inclusion of a small $(1-3 \mathrm{~mm})$ margin of normal tissue in the lateral margins. For extensive lesions ( $>40 \mathrm{~mm}$ or those that occupied $>1$ wall of the colon), injection and resection of each wall or a half segment was performed sequentially to allow free access to tissue within potentially sharp angulations, to enhance visualization, and to minimize time for subsidence of the fluid cushion due to diffusion. Ablative techniques were avoided and only used sparingly when complete resection was not possible. "Soft coagulation" to treat the lateral margins of piecemeal resected lesions was undertaken once its role in reducing recurrence was recognized [18]. Complete resection of the lesion was defined histologically as clear margins at the resection border. Histological assessment was carried out on excised and retrieved tissue.

Immediate bleeding was defined as that which required additional measures above dilute epinephrine $(1: 10000)$. This 
- Table 1 Patient demographics, lesion characteristics and dysplasia for the EMR and surgery cohorts.

\begin{tabular}{|c|c|c|c|}
\hline & EMR cohort $(n=183)$ & Surgery cohorts $(n=64)$ & $P$ value \\
\hline Age, median (IQR), years & $68(15.8)$ & $68(17.3)$ & 0.697 \\
\hline \multicolumn{4}{|l|}{ Sex, n (\%) } \\
\hline - Male & $109(59.6)$ & $31(48.4)$ & $<0.001$ \\
\hline - Female & $74(40.4)$ & $33(51.6)$ & \\
\hline Lesion size, n (\%) & $n=216$ & $n=69$ & \\
\hline . $<15$ mm & $32(14.8)$ & $20(29.0)$ & 0.008 \\
\hline - $15-30 \mathrm{~mm}$ & $138(63.9)$ & $21(30.4)$ & $<0.001$ \\
\hline - $30.1-40 \mathrm{~mm}$ & $28(13.0)$ & $11(15.9)$ & 0.023 \\
\hline - $>40 \mathrm{~mm}$ & $18(8.3)$ & $13(18.8)$ & 0.015 \\
\hline - Not recorded & $0(0.0)$ & $4(5.8)$ & 0.531 \\
\hline Location of lesions, n (\%) & $n=216$ & $n=69$ & \\
\hline - Rectum & $16(7.4)$ & $4(5.8)$ & 0.727 \\
\hline - Rectosigmoid junction & $3(1.4)$ & $0(0.0)$ & 0.325 \\
\hline - Sigmoid colon & $30(13.9)$ & $6(8.7)$ & 0.031 \\
\hline - Descending colon & $13(6.0)$ & $3(4.3)$ & 0.600 \\
\hline - Transverse colon & $33(15.3)$ & $7(10.1)$ & 0.166 \\
\hline - Hepatic flexure & $12(5.6)$ & $6(8.7)$ & 0.025 \\
\hline - Ascending colon & $64(39.6)$ & $29(42.0)$ & 0.019 \\
\hline - Cecum & $45(20.8)$ & $14(20.3)$ & 0.003 \\
\hline Paris classification, n (\%) & $\mathrm{n}=216$ & & \\
\hline . $0-$ Is & $66(30.6)$ & NA & \\
\hline . $0-11 a$ & $75(34.7)$ & NA & \\
\hline . $0-1 \mathrm{~s}+0-11 \mathrm{a}$ & $42(19.4)$ & NA & \\
\hline . $0-11 a+0-I I b$ & $4(1.9)$ & NA & \\
\hline - Other & $29(13.4)$ & NA & \\
\hline \multicolumn{4}{|l|}{ Post-procedural bleeding, n (\%) } \\
\hline - No & $172(94.0)$ & NA & \\
\hline - Yes & $11(6.0)$ & NA & \\
\hline \multicolumn{4}{|l|}{ En bloc resection, n (\%) } \\
\hline - No & $122(56)$ & NA & \\
\hline - Yes & $94(44)$ & NA & \\
\hline \multicolumn{4}{|l|}{ Lesion histology, n (\%) } \\
\hline - Tubular adenoma & $136(63.0)$ & $15(23)$ & $<0.001$ \\
\hline - Tubulovillous/villous adenoma & $43(20.0)$ & $21(33)$ & 0.055 \\
\hline - Sessile serrated adenoma/polyp & $36(14.8)$ & $7(11)$ & 0.649 \\
\hline - Adenocarcinoma & $5(2.3)$ & $4(6)$ & 0.150 \\
\hline - Normal bowel mucosa & 0 & $17(27)$ & $<0.001$ \\
\hline \multicolumn{4}{|l|}{ Dysplasia, n (\%) } \\
\hline - None & $48(22.2)$ & $19(27.5)$ & $<0.001$ \\
\hline - Low grade & $108(50.0)$ & $34(49.3)$ & 0.438 \\
\hline
\end{tabular}


Table 1 (Continuation)

\begin{tabular}{|l|c|c|c|}
\hline & EMR cohort $(\mathbf{n}=\mathbf{1 8 3})$ & Surgery cohorts $(\mathbf{n}=\mathbf{6 4})$ & \multicolumn{1}{|c|}{$\mathbf{P}$ value } \\
\hline - High grade & $49(22.7)$ & $16(23.2)$ & 0.820 \\
\hline - Carcinoma in situ & $6(2.8)$ & $0(0.0)$ & $<0.001$ \\
\hline - Adenocarcinoma & $5(2.3)$ & $0(0.0)$ & $<0.001$ \\
\hline
\end{tabular}

type of bleeding was primarily managed by the placement of endoscopic clips (Resolution Clip, Boston Scientific, Natick, Massachusetts, United States; QuickClip, Olympus). Bleeding was classified as delayed if it occurred after discharge and was of a clinically significant level requiring readmission.

Following EMR, all patients were observed for 4 hours. Clinical follow-up occurred 2 weeks post EMR, when results were communicated to the referring specialists and patients. Grading of dysplasia was reported according to the revised Vienna classification [19]. In patients who underwent a technically successful EMR, with complete resection and without submucosal invasive cancer (SMIC), or High Grade Dysplasia (HGD), colonoscopy at 12 months was recommended and these were commonly performed by the referring endoscopist. For patients with HGD or a potentially incomplete resection, repeat endoscopy was scheduled at 4 months by the senior investigator. When invasive malignancy was detected, surgical review was advised. At the follow-up colonoscopy, the site of the EMR scar was visualized and if required, biopsies were taken. Residual and/or recurrent adenoma was excised using a small, thin-wire snare and, if necessary, ablated with argon plasma coagulation (APC). All specimens were retrieved and reviewed by expert gastrointestinal pathologists.

\section{Statistical analysis}

Statistical analyses were performed using IBM SPSS Version 25.0 (Armonk, NY, United States: IBM Corp.). All averages are expressed as mean and standard deviation for normally distributed data or median and interquartile range for non-normally distributed data. Chi-squared testing was used to compare proportions. The $t$ test was used to compare means.

\section{Results}

Between January 2009 and January 2018, 194 consecutive patients were enrolled for EMR. However, EMR was not attempted in eight patients due to lesion appearance being suggestive of invasive malignancy or technical unfeasibility (extending over the dentate line or involving the ileocecal valve with extension into the terminal ileum). In the same period, 32 patients were referred for surgery including three patients in whom EMR was unsuccessful. The remaining 29 patients had surgery performed in lieu of EMR due to referrer or surgeon unfamiliarity with availability and efficacy of EMR for removal of non-malignant polyps. Therefore, a total of 183 patients (194-11 patients) underwent successful EMR (109 male [60\%], median age 68 years, range $25-89$ years). Of these, 159 had a single lesion, 16 had 2 lesions, 7 had 3 lesions, and 1 had 4 lesions removed by EMR, accounting for 216 lesions in total. Details are presented in $>$ Table 1.

Over the 4-year period before the introduction of EMR, 32 surgical procedures were performed for 35 benign colonic lesions (16 male [50\%], median age 68 years, range 27-84 years). In the 9 years following the introduction of a dedicated EMR service, 32 patients with 33 benign lesions were referred for surgery (15 male [47\%], median age 70 years, range $35-$ 88 years). Surgery type is presented in $>$ Table 2 .

In the period 2009 to 2017, the mean size of lesion removed by EMR was $24 \mathrm{~mm}$ (range $15-80 \mathrm{~mm}$ ). In the period from 2009 to 2017, polyps removed by SR compared to EMR were larger (30 mm, range $5-70 \mathrm{~mm}, P=0.003$ ). There was no statistically significant difference in polyp size between those removed by SR in the period from 2004 to 2008 and EMR resected polyps in the period 2009 to $2017(25 \mathrm{~mm}$, range $5-80 \mathrm{~mm}, P=$ 0.086).

Of the 107 EMR cases with 4 months of follow-up data, colonoscopy revealed no recurrence in 100 cases $(93.5 \%)$ and recurrence in 7 cases (6.5\%). Data was not available in 76 cases (41.5\%) due to follow-up colonoscopy being performed at an external referring institution, patients declining repeat colonoscopy, patient death (unrelated to EMR), or definitive colorectal surgery. All seven cases of recurrence were completely treated during follow-up colonoscopy and there were no cases of progression of recurrent disease to malignancy.

\section{Length of stay and complications}

Median LoS post-colorectal surgery in the 2005-2008 cohort was 10 days (IQR 8.25, range 3-65 days), and in the 20092017 cohort, median LoS was 8.5 days (IQR 8, range 3-30 days). The median LoS after all colorectal surgery was 9 days (IQR 9.25 days), and was significantly longer than LoS associated with EMR (median 1 day, IQR $0, P<0.0001$ ). The LoS per lesion removed was significantly longer before the introduction of an EMR service (13.0 days) compared to following its introduction ( 2.7 days, $P<0.001$ ).

There were no deaths in the peri-procedural setting in either the EMR or colorectal surgery cohort. Of the 64 surgical procedures performed during the entire study period (2004-2017), 35 (54.7\%) were associated with major peri-operative complications in a total of 51 major complications. In total, 14 complications were seen associated with the 183 EMR procedures (7.7\%). Delayed bleeding occurred in 11 cases, all of which 
- Table 2 Type of surgery used in the surgery cohorts for the periods before and after initiation of the EMR service.

\begin{tabular}{|l|c|c|}
\hline Surgery type & $\mathbf{2 0 0 5 - 2 0 0 8}$ & $\mathbf{2 0 0 9 - 2 0 1 7}$ \\
\hline Abdominoperineal resection, $\mathrm{n}(\%)$ & $2(6.3)$ & $1(3.1)$ \\
\hline Anterior resection, $\mathrm{n}(\%)$ & $1(3.1)$ & $2(6.3)$ \\
\hline lleocolic resection, $\mathrm{n}(\%)$ & $1(3.1)$ & $1(3.1)$ \\
\hline Left hemicolectomy, $\mathrm{n}(\%)$ & $2(6.3)$ & $1(3.1)$ \\
\hline Right hemicolectomy, $\mathrm{n}(\%)$ & $23(71.9)$ & $25(78.1)$ \\
\hline Sigmoid colectomy, $\mathrm{n}(\%)$ & $1(3.1)$ & $1(3.1)$ \\
\hline Subtotal colectomy, $\mathrm{n}(\%)$ & $2(6.3)$ & $1(3.1)$ \\
\hline Total, n & 32 & 32 \\
\hline
\end{tabular}

were managed either conservatively or with transfusion of packed red blood cells. Operative management was not required. Perforation occurred on two occasions, both episodes managed conservatively with broad-spectrum antibiotics. One procedure was complicated with aspiration pneumonitis requiring a 3-day admission. The complication rate associated with EMR was significantly less than that associated with colorectal surgery, $P<0.0001$ ( $\vee$ Table 3 ).

\section{Financial impact}

The median WIES funding associated with each EMR was 0.3 units (IQR 0.2) compared with the median WIES of 4.6 units (IQR 2.5) for SR. EMR, including readmissions and management of complications or failed procedures, had a WIES unit per lesion removed rate of 0.46 . SR, including management of associated complications, had a significantly greater WIES unit per lesion removed rate of $5.2(P<0.001)$.

\section{Discussion}

EMR is an effective technique for the removal of large sessile or laterally spreading colonic lesions not amenable to removal by standard endoscopic means. The introduction of an EMR service to our regional institution reduced the need for colorectal surgical procedures and hence reduced the total cost per lesion removed, complications, and LoS.

The average WIES value of 0.3 for EMR is significantly less than the average colorectal surgery value of 4.6. This decrease of 4.3 WIES units is equivalent to a saving of AUD $\$ 19543.5$ per procedure. In addition to this significant cost saving, the provision of a non-metropolitan EMR service likely provides many other less tangible financial benefits, such as reduced travel costs, accommodation costs, and in some cases, decreased productivity loss with earlier return to work. Finally, many rural residents are resistant to transfer and management at metropolitan centers, and provision of EMR in a non-metropolitan center may capture those patients who would otherwise have elected for surgical management performed in a local setting, or even avoided healthcare entirely.
Table 3 Complications in the EMR and surgery cohorts.

\begin{tabular}{|l|l|l|}
\hline Complications & $\begin{array}{l}\text { EMR cohort } \\
(\mathbf{n = 1 8 3 )}\end{array}$ & $\begin{array}{l}\text { Surgery cohorts } \\
(\mathbf{n}=\mathbf{6 4})\end{array}$ \\
\hline lleus & 0 & 7 \\
\hline Pulmonary embolism & 0 & 2 \\
\hline $\begin{array}{l}\text { Non-ST elevation myocardial } \\
\text { infarction }\end{array}$ & 0 & 2 \\
\hline $\begin{array}{l}\text { Anastomosis complications } \\
\text { including wound infection, } \\
\text { post-procedure bleeding }\end{array}$ & 11 & 12 \\
\hline $\begin{array}{l}\text { Other infection } \\
\text { Others (includes stroke, } \\
\text { delirium, perforation) }\end{array}$ & 3 & 14 \\
\hline $\begin{array}{l}\text { Total cases with one or more } \\
\text { complication), } n \text { (\%) }\end{array}$ & $14(7.7)$ & 14 \\
\hline
\end{tabular}

The complication rate associated with EMR has been described across several series as between $4 \%$ and $7 \%$ [7-9]. Our complication rate of $7.7 \%$ is consistent with these previously published findings. Average LoS was significantly less for patients undergoing EMR compared to colorectal surgical procedures. This is intrinsically linked to complication rate; a study reviewing complication rates following elective surgery for non-malignant colorectal polyps found a 30-day mortality of $0.7 \%$, a major postoperative adverse event rate of $14 \%$, and a readmission rate of $7.8 \%$, while the need for a second major surgery was $3.6 \%[14]$.

Determination of long-term success following EMR requires data on recurrence and/or progression. Our recurrence rate $(6.5 \%)$ at 4 -month follow-up colonoscopy was less than that quoted in the literature, which ranged from $15 \%$ to $47 \%$ [4, $13,20-24]$. The low recurrence rate in our series is likely to be due to the high en bloc resection rate and the use of soft tip coagulation after piecemeal resections. There were five instances where EMR histology had the unexpected finding of adenocarcinoma. All five cases proceeded to surgical management.

Ours is the first series to examine the impact of EMR performed in a regional center. The introduction of a dedicated EMR service resulted in a significant decrease in the number of patients with benign colonic lesions undergoing surgery. As our series demonstrates, appropriately performed EMR has many advantages over colorectal surgery consistent with published literature; in particular, lower morbidity $[5,13]$, lower complication rate $[10,11]$, and reduced length of stay [10]. Despite these clear benefits, many patients are still referred for surgery for resection of EMR appropriate lesions [25]. Explanations encountered during chart review of the patients undergoing surgery include the erroneous belief that a lesion is too large to be resectable endoscopically or that a lesion is malignant despite endoscopic evidence or biopsies indicating otherwise. Other reasons may include the views that lesions removed via EMR technique have a high risk of recurrence and that EMR is therefore not a definitive form of therapy. In some instances, there is 
also lack of awareness or training in accurate endoscopic assessment of lesions. There are potentially other entrenched biases against referring patients for EMR, a lack of familiarity with EMR, and either a real or perceived dearth of EMR services.

Our series helps address some of these issues, suggesting that EMR can be safely performed in a non-metropolitan setting. Finally, EMR does not preclude patients from having definitive surgery if carcinoma-in situ/adenocarcinoma is found, or if a lesion is endoscopically non-resectable [26].

This study is subject to some limitations. Firstly, while EMR data were collected prospectively, surgical data were collected retrospectively with a chart review. However, two researchers completed the chart review, thus reducing the chance of inaccurate data collection. Secondly, this study investigated the introduction of EMR to a single regional center with a single operator. The general applicability of results to metropolitan centers is uncertain. Between 2004 and 2008, our data assumed that all patients with lesions not amenable to traditional colonoscopic removal were managed with colorectal surgery at our study institution. We do not have data with regard to any patients who might have had lesions assessed as not appropriate for traditional colonoscopic removal and who may have been subsequently referred to a metropolitan center for EMR. Furthermore, calculation of WIES funding includes the index admission, future admissions for management of complications, and follow-up colonoscopies. Numbers of outpatient clinic appointments, future surveillance colonoscopies, medications, and other indefinable costs were not included in calculations of cost and may affect the accuracy of estimates of cost savings.

In conclusion, this study demonstrates that a dedicated EMR service results in a significant reduction in the total number of surgical procedures performed for the primary indication of removing large sessile or laterally spreading colonic lesions. In the current series, complication rates were consistent with metropolitan counterparts when performed by an expert endoscopist. Compared to traditional surgical management, EMR was associated with a decreased average length of hospital stay ( 9 days vs 1 day, $P<0.0001$ ) and decreased complication rates ( $54.7 \%$ vs $7.7 \%, P<0.0001)$. There was a cost saving of approximately AUD\$19 543.5 per patient treated with EMR rather than surgery. Despite increasing awareness of the clinical and financial advantages of EMR, not all patients with endoscopically resectable lesions are referred for EMR. The barriers to further uptake of EMR require further investigation.

\section{Competing interests}

None

\section{References}

[1] Raju GS, Lum PJ, Ross WA et al. Outcome of EMR as an alternative to surgery in patients with complex colon polyps. Gastrointest Endosc 2016; 84: 315-325
[2] Winawer S], Zauber AG, Ho MN et al. The National Polyp Study Workgroup. Prevention of colorectal cancer by colonoscopic polypectomy. NEJM 1993; 329: 1977 - 1981

[3] Saito Y, Yamada M, So E et al. Colorectal endoscopic submucosal dissection: Technical advantages compared to endoscopic mucosal resection and minimally invasive surgery. Dig Endosc 2014; 26: (Suppl. 01): $52-61$

[4] Bories E, Pesenti C, Monges G et al. Endoscopic mucosal resection for advanced sessile adenoma and early-stage colorectal carcinoma. Endoscopy 2006; 38: 231 - 235

[5] Swan MP, Bourke M], Alexander S et al. Large refractory colonic polyps: is it time to change our practice? A prospective study of the clinical and economic impact of a tertiary referral colonic mucosal resection and polypectomy service (with videos). Gastrointest Endosc 2009; 70: $1128-1136$

[6] Jameel JK, Pillinger SH, Moncur P et al. Endoscopic mucosal resection $(\mathrm{EMR})$ in the management of large colo-rectal polyps. Colorectal Dis 2006; 8: 497-500

[7] Sawhney MS, Salfiti N, Nelson DB et al. Risk factors for severe delayed postpolypectomy bleeding. Endoscopy 2008; 40: 115-119

[8] Burgess NG, Metz AJ, Williams S] et al. Risk factors for intraprocedural and clinically significant delayed bleeding after wide-field endoscopic mucosal resection of large colonic lesions. Clin Gastroenterol Hepatol 2014; 12: 651-661.e1-3

[9] Masci E, Viale E, Notaristefano C et al. Endoscopic mucosal resection in high- and low-volume centers: a prospective multicentric study. Surg Endosc 2013; 27: 3799-3805

[10] Lezoche E, Feliciotti F, Paganini AM et al. Laparoscopic vs open hemicolectomy for colon cancer. Surg Endosc 2002; 16: 596 - 602

[11] Nelson H, Sargent DJ, Wieand HS et al. Clinical Outcomes of Surgical Therapy Study Group.. A comparison of laparoscopically assisted and open colectomy for colon cancer. NEJM 2004; 350: 2050 - 2059

[12] Billeter AT, Polk HC Jr, Hohmann SF et al. Mortality after elective colon resection: the search for outcomes that define quality in surgical practice. J Am Coll Surg 2012; 214: 436 - 443; discussion 443-444

[13] Moss A, Bourke MJ, Williams SJ et al. Endoscopic mucosal resection outcomes and prediction of submucosal cancer from advanced colonic mucosal neoplasia. Gastroenterology 2011; 140: 1909-1918

[14] van Nimwegen LJ, Moons LMG, Geesing JMJ et al. Extent of unnecessary surgery for benign rectal polyps in the Netherlands. Gastrointest Endosc 2018; 87: 562 - 570.e1

[15] Manfredi S, Piette C, Durand G et al. Colonoscopy results of a French regional FOBT-based colorectal cancer screening program with high compliance. Endoscopy 2008; 40: $422-427$

[16] Bronzwaer MES, Koens L, Bemelman WA et al. Volume of surgery for benign colorectal polyps in the last 11 years. Gastrointest Endosc 2018; 87: 552 - 561.e1

[17] Klein A, Bourke MJ. How to perform high-quality endoscopic mucosal resection during colonoscopy. Gastroenterology 2017; 152: 466 471

[18] Klein A, Jayasekeran V, Hourigan LF et al. 812b A multi-center randomized control trial of thermal ablation of the margin of the post endoscopic mucosal resection (EMR) mucosal defect in the prevention of adenoma recurrence following EMR: Preliminary results from the "SCAR" study. Gastroenterology 2016; 150: S1266-S1267

[19] Dixon MF. Gastrointestinal epithelial neoplasia: Vienna revisited. Gut 2002; $51: 130-131$

[20] Jang ES, Kim JW, Jung Y] et al. Clinical and endoscopic predictors of colorectal adenoma recurrence after colon polypectomy. Turk J Gastroenterol 2013; 24: 476-482

[21] Tate DJ, Desomer L, Klein A et al. Adenoma recurrence after piecemeal colonic EMR is predictable: the Sydney EMR recurrence tool. Gastrointest Endosc 2017; 85: 647-656 
[22] Briedigkeit A, Sultanie O, Sido B et al. Endoscopic mucosal resection of colorectal adenomas $>20 \mathrm{~mm}$ : Risk factors for recurrence. World J Gastrointest Endosc 2016; 8: 276 - 281

[23] Lim TR, Mahesh V, Singh S et al. Endoscopic mucosal resection of colorectal polyps in typical UK hospitals. World J Gastroenterol 2010; 16: $5324-5328$

[24] Ortiz AM, Bhargavi P, Zuckerman M] et al. Endoscopic mucosal resection recurrence rate for colorectal lesions. South Med J 2014; 107: $615-621$
[25] Gomez V, Racho RG, Woodward TA et al. Colonic endoscopic mucosal resection of large polyps: Is it safe in the very elderly? Dig Liver Dis 2014; 46: 701 - 705

[26] Rickert A, Aliyev R, Belle S et al. Oncologic colorectal resection after endoscopic treatment of malignant polyps: does endoscopy have an adverse effect on oncologic and surgical outcomes? Gastrointest Endosc 2014; 79: 951 - 960 